\title{
Airway structure and inflammatory cells in fatal attacks of asthma
}

\author{
N. Carroll*, S. Carello**, C. Cooke+, A. James*
}

Airway structure and inflammatory cells in fatal attacks of asthma. N. Carroll, S. Carello, C. Cooke, A. James. CERS Journals Ltd 1996.

ABSTRACT: Fatal attacks of asthma usually occur against a background of chronic persistent symptoms, presumably due to chronic airway inflammation and changes in airway wall structure. Death from asthma is usually attributed to excessive airway narrowing due to a combination of muscle spasm and mucous plugging. To test the hypothesis that airway wall structure and/or the inflammatory cell profile are related to the duration of a fatal attack of asthma, inflammatory cell profiles and airway structure were examined in cases of fatal asthma and related to the duration of the fatal attack.

In transverse sections of large and small airways from subjects dying from asthma, the numbers of eosinophils, neutrophils and lymphomononuclear cells were counted. The amount of smooth muscle shortening, the areas of airway wall, smooth muscle, mucous gland and cartilage were measured. Cell counts, airway dimensions and muscle shortening were compared in cases dying within $2 \mathrm{~h}$ of the fatal attack (short duration) and those dying more than $5 \mathrm{~h}$ after the onset of the fatal attack (long duration).

In cases with fatal attacks of short duration, the numbers of neutrophils and the mucous gland area were increased and the numbers of eosinophils were reduced compared to cases with fatal attacks of long duration. Lymphocyte numbers, airway wall thickness, the areas of smooth muscle and cartilage and the amount of smooth muscle shortening were similar in the two groups.

These findings suggest fatal attacks of asthma may be triggered by an inflammatory stimulus and suggest that increased production of mucous may contribute to sudden death in such cases.

Eur Respir J., 1996, 9, 709-715.
*Dept of Pulmonary Physiology, Sir Charles Gairdner Hospital, Nedlands, Western Australia. **Dept of Pathology, University of Western Australia. +Dept of Forensic Pathology, State Health Laboratories, QEII Medical Center, Perth, Australia.

Correspondence: A. James

Dept of Pulmonary Physiology

Sir Charles Gairdner Hospital

Verdun St

Nedlands 6009

Western Australia

Keywords: Fatal asthma inflammation morphology

Received: May 221995

Accepted after revision December 271995
Mortality rates for asthma in Australia have recently been 1.5 per 100,000 population in the 5-34 year age group and as high as 1.9 per 100,000 population in New Zealand [1]. Recent figures suggest this rate may be falling [2]. Studies of fatal and near fatal attacks of asthma [3-8] have identified a number of risk factors for death from asthma, including a previous history of severe or life-threatening attacks, previous hospital admissions, attendance at emergency departments for asthma, failure to seek or maintain medical care, and psychosocial factors, such as depression and family conflict. The New Zealand Asthma Mortality Study [4] suggested that poor self-assessment of asthma severity with a tendency to self-care, poor compliance with therapy, and inappropriate treatment, particularly in younger patients, also added to the risk of death from asthma. Although sudden and unexpected death in patients with apparently mild and well-controlled asthma has been reported [9], the studies of risk factors suggest that death from asthma most often occurs in the setting of undertreated and unrecognized asthma.

The epidemiological and clinical data are supported by postmortem studies of the airways in cases of fatal asthma [10-17]. The presence in such cases of extensive bronchial epithelial damage, plugging of the airway lumen with mucus and cellular debris, thickening of the basement membrane, airway smooth muscle hypertrophy and hyperplasia, mucous gland hyperplasia, and infiltration of the airway wall with inflammatory cells, suggests longstanding, persistent airway inflammation and structural change. These pathological changes are associated with thickening of the airway wall both in large and small airways, to a degree which could account for the excessive airway narrowing observed in asthma [16, 18].

It seems likely, therefore, that patients with longstanding disease and altered airway structure are more likely to die from asthma. The mechanisms of death in such cases remain unclear. Contributing factors might include increased airway wall thickness, muscle shortening and mucus secretion into the lumen. The triggering factors for the fatal attack, which may be quite sudden, are also unclear. An association between exposure to the mould Alternaria and near fatal attacks of asthma has been reported [19]. Whether such stimuli act via smooth muscle shortening, secretion of mucus, acute inflammatory exudation and infiltration or a combination of these events remains unknown.

Azzawi et al. [17] have reported that the number of activated eosinophils was significantly higher in subjects 
in whom the duration of the terminal attack of asthma was in excess of $24 \mathrm{~h}$ compared with those who died suddenly. In a subsequent study, SuR et al. [20] reported that airways from people who died within $1 \mathrm{~h}$ of the onset of asthma symptoms ("sudden onset fatal asthma") had more neutrophils and relatively less eosinophils than people whose death occurred more than $2.5 \mathrm{~h}$ after the initial onset of asthma symptoms ("slow onset fatal asthma"). They also found that the number of eosinophils correlated directly, and the number of neutrophils correlated indirectly, with the duration of the terminal asthma attack. They suggested that, in fact, these two groups of patients dying from asthma (i.e. "sudden and slow") may represent two distinct pathological entities initiated by different inflammatory stimuli and resulting in distinct mechanisms of excessive airway narrowing.

It might also be argued that the different profiles of inflammatory cells in relation to time in the cases of fatal asthma may simply represent the progression of inflammatory events after exposure to a single, powerful stimulus. The duration of the fatal attack may be determined by pre-existing airway structure, such as area of smooth muscle, mucous glands or airway wall thickness. It was, therefore, hypothesized that if the duration of survival during a fatal attack of asthma is related to the nature of the inflammatory cell infiltrate and airway structure, that these features will be related to the duration of the terminal episode of asthma.

To test this hypothesis, inflammatory cell profiles, airway wall thickness and the areas of the components of the airway wall were measured and compared in cases of fatal asthma categorized by the time interval from onset of asthma attack to death.

\section{Methods}

\section{Cases}

The records of 18 cases of fatal asthma coming to Coroner's autopsy were examined. From these, the time interval from the onset of the fatal attack until death could be determined in eight cases. All deaths occurred out of hospital. In all cases, asthma was given as the cause of death at autopsy, the report of events prior to death was consistent with asthma, cardiovascular and intracranial causes of death were excluded and a history of asthma was obtained from relatives, medical practitioner or hospital files. Information regarding the immediate events prior to death was obtained from Coroner's files. Based on duration of the final asthma episode, the cases were arbitrarily categorized into two groups: those where death occurred within $2 \mathrm{~h}(\mathrm{n}=4)$; and those where death occurred after $5 \mathrm{~h}(\mathrm{n}=4)$.

\section{Histological preparation and tissue sampling}

Following fixation, specimens of all identifiable lobar, segmental and subsegmental airways which could be cut in transverse section and were free from branching were obtained in each case. Six parenchymal sections were taken at random from the midsaggital slice and all sections were processed through a series of graded alcohols

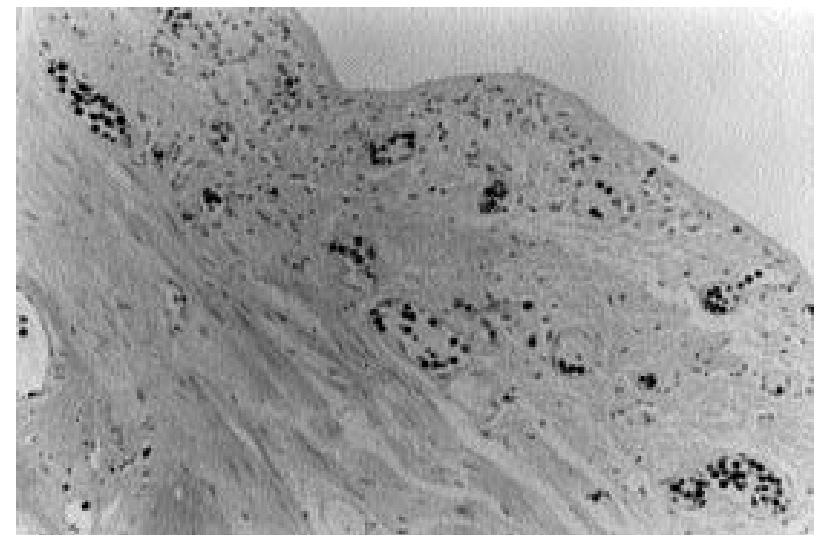

Fig. 1. - Submucosa from a transverse cartilaginous airway stained with anti-neutrophil elastase from a case of short duration fatal asthma. The epithelium is absent and the basement membrane is prominent (open arrow heads). Many neutrophils are seen between the airway smooth muscle and the basement membrane. Note the large numbers of neutrophils within blood vessels (closed arrows). Scale bar $=50 \mu \mathrm{m}$ (Magnification $\times 400$ ).

and embedded in paraffin wax, sectioned $(5 \mu \mathrm{m})$ and stained with haematoxylin and eosin and with an antineutrophil elastase monoclonal antibody (Dako Patts, Copenhagen) to identify neutrophils (fig. 1). Briefly, tissue sections were deparaffinized with xylene and placed in graded alcohol solutions from absolute alcohol down to water. Endogenous peroxidase activity was blocked by immersion in 3\% hydrogen peroxide in water for 5 min. Nonspecific attachment of primary antiserum was prevented by pretreatment with $5 \%$ normal swine serum for $20 \mathrm{~min}$. The primary antiserum (neutrophil elastase) was applied to sections and incubated overnight in a humidity chamber at $4^{\circ} \mathrm{C}$. After washing in tris-buffered saline (TBS), the sections were incubated with biotinylated, (goat) anti-mouse immunoglobulin G (IgG) (Silenus Laboratories, Hawthorn, Victoria, Australia). After 45 min the sections were washed in TBS and incubated with streptavidin-peroxidase complex (Silenus) and incubated for another $45 \mathrm{~min}$. After washing, colour was developed

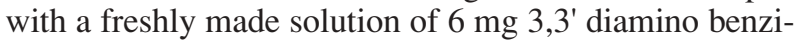
dine tetrahydrochloride (Sigma) in $10 \mathrm{~mL}$ of TBS with 10 $\mu \mathrm{L}$ of $30 \%$ hydrogen peroxide for $8 \mathrm{~min}$. Sections were then washed and counterstained with Gill's haematoxylin, dehydrated in alcohol, cleared in xylene and mounted in DEPEX.

\section{Measurements}

Using a light microscope, camera lucida and digitizing tablet connected to a mini-computer, measurements were made on airways that were cut in transverse section, defined by even thickness of mucosa and submucosa. The internal area $\left(\mathrm{Ai}_{\mathrm{i}}\right)$ and perimeter $(\mathrm{Pi})$ were defined by the luminal border of the airway epithelium in airways where more than $50 \%$ of the epithelium was intact. Where portions of the basement membrane were not covered by epithelium, the border was interpolated between two consecutive, intact areas of epithelium; the area (Abm) and perimeter $(\mathrm{Pbm})$ defined by the basement membrane; the area (Amo) and perimeter (Pmo) defined by the outer border of the airway smooth muscle and the total area (Ao) and perimeter $\left(\mathrm{Po}_{0}\right)$ defined by the adventitial surface of the airway were measured. Where the airway 
was too large to be viewed entirely using a $\times 4$ objective lens on the microscope, the slide was enlarged using a Nikon Profile Projector (Model 6C; Nippon, Kogaku, Japan) and the image was traced onto transparent plastic sheets for measurement on the digitizing tablet. The total areas of mucous gland, smooth muscle and cartilage were measured in each airway with the digitizer.

\section{Calculations}

The airway wall areas and the areas of cartilage, mucous gland and smooth muscle were expressed as area $\mathrm{mm}^{-1}$ of basement membrane. Compartmental areas of the airway wall were calculated as follows: the total wall area $(\mathrm{WAtot}=\mathrm{Ao}-\mathrm{Abm})$ and the inner wall area $(\mathrm{WAi}=\mathrm{Amo}$ - Abm) were calculated by subtracting the area defined by the basement membrane from the total area and muscle areas, respectively; and the outer wall area (WAo = Ao - Amo) was calculated by subtracting the outer area from the total area. To calculate the percentage muscle shortening (PMS) present in each airway, "relaxed" airway dimensions were derived using the basement membrane perimeter $(\mathrm{Pbm})$ and the inner wall area, since these dimensions are independent of smooth muscle tone and lung volume [21]. The relaxed lumen area $(\mathrm{Abm}, \mathrm{r})$ is a circle with a circumference equal to the measured basement membrane perimeter $(\mathrm{Pbm})$; thus, $\mathrm{Abm}, \mathrm{r}=(\mathrm{Pbm})^{2} / 4 \pi$. By adding the measured area of wall between the basement membrane and outer muscle border (Amo-Abm) to Abm,r the relaxed outer area (Amo,r) was obtained. The percentage of smooth muscle shortening was determined by comparing the observed muscle "length", given by the outer perimeter $\left(\mathrm{P}_{\mathrm{o}}\right)$, to its calculated, relaxed length

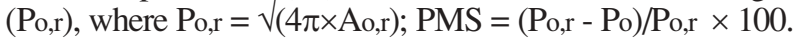

\section{Cell counts}

In tissue sections stained with haematoxylin and eosin, the total number of eosinophils, neutrophils and lymphomononuclear cells and in tissue sections stained with anti-neutrophil elastase, only neutrophils were counted from the basement membrane to the outer border of the airway smooth muscle (i.e. inner wall area). Cells were not counted if they were in mucous glands and cell numbers were expressed as cells $\cdot \mathrm{mm}^{-1}$ of $\mathrm{Pbm}$. Eosinophils were identified using morphological criteria - cells with granular eosinophilic cytoplasm and with two nuclear lobules. Cells with eosinophilic nongranular cytoplasm with more than two nuclear lobules were categorized as neutrophils. All lymphomononuclear cells were counted in membranous airways and the cell numbers were expressed cells $\cdot \mathrm{mm}^{-1}$ of $\mathrm{Pbm}$. In large airways, the numbers of lymphomononuclear cells between the basement membrane and the outer border of the airway smooth muscle were counted in at least eight randomly chosen high-power fields (HPF) using a calibrated graticule eyepiece. The area of tissue in which lymphomononuclear cells were counted was measured for each HPF and the mean number of cells $\cdot \mathrm{mm}^{-2}$ was calculated. Preliminary work had shown that the cumulative mean cell count varied by less than $5 \%$ after measurement of eight random fields. The mean lymphomononuclear cell number $\cdot \mathrm{mm}^{-2}$ was then multiplied by the total inner wall area to calculate the total number of lymphomononuclear cells, and this number was then divided by the $\mathrm{Pbm}$ to obtain the number of cells $\cdot \mathrm{mm}^{-1} \mathrm{Pbm}$. The majority of lymphomononuclear cells were identified as lymphocytes using morphological criteria - dark, even-staining, small nuclei with scanty cytoplasm. Identification of lymphocytes was confirmed in a preliminary study [22] using the leucocyte common antigen (LCA) monoclonal antibody as a pan-leucocyte marker, UCHL1 antibody as a marker of a "primed" T-cell subset, and L26 as a specific B-cell marker. Very few B-cells were seen. Additional confirmation using morphological criteria was obtained by interobserver comparisons with pathologists and cytologists experienced in observation of pulmonary tissue. In airways stained with neutrophil elastase, all positively stained cells were counted between the basement membrane and smooth muscle layer and it was also noted whether the cell was in a vessel or not. Extracellular deposition of elastase was not quantitated.

\section{Data analysis}

The absolute cell counts were logarithmically transformed. For each case, the mean eosinophil, neutrophil and lymphocyte counts $\cdot \mathrm{mm}^{-1} \mathrm{Pbm}$ were calculated only for airways with a $\mathrm{Pbm}$ greater than $10 \mathrm{~mm}$, corresponding to midsized and large cartilaginous airways. Structural dimensions were measured and the PMS was calculated in four arbitrary airway size groups to represent membranous bronchioles $(\mathrm{Pbm}<4 \mathrm{~mm})$, subsegmental airways (Pbm 4-10 mm); segmental airways ( $\mathrm{Pbm}>10-18$ $\mathrm{mm})$; and lobar airways $(\mathrm{Pbm}>18 \mathrm{~mm})$. The mean cell counts and structural dimensions from each case within each size group were combined and the grand mean $( \pm \mathrm{SD})$ for that size group was calculated for case groups. Comparisons between case groups (short duration and long duration) for cell counts were made using the MannWhitney U-test and for structural dimensions using a one-way analysis of variance (ANOVA). A p-value of less than 0.05 was considered to be significant.

\section{Results}

\section{Cases}

Subject characteristics are shown in table 1. The age (mean \pm SD) in long duration cases $(42 \pm 10 \mathrm{yrs})$ was higher than in short duration cases $(27 \pm 21 \mathrm{yrs})$, although there was considerable overlap. The duration of the final asthma attack (mean $\pm \mathrm{SD}$ ) was $1.2 \pm 0.4 \mathrm{~h}$ in short duration cases compared with $9.3 \pm 4.2 \mathrm{~h}$ in long duration cases. Severity of asthma based on type and frequency of medications used, duration of asthma, symptom frequency and hospitalizations due to asthma was similar in each group. There were no current smokers in either case group. An average of 19 sections of large airways or parenchyma were obtained and at least 15 airways (range 15-27) were measured for each subject. 
Table 1. - Subject characteristics

\begin{tabular}{lcc}
\hline & $\begin{array}{c}\text { Short duration } \\
(\mathrm{n}=4)\end{array}$ & $\begin{array}{c}\text { Long duration } \\
(\mathrm{n}=4)\end{array}$ \\
\hline Sex M/F & $2 / 2$ & $1 / 3$ \\
Age yrs & $27 \pm 21$ & $42 \pm 10$ \\
Duration of final asthma & & \\
$\quad$ attack $\mathrm{h}^{\#}$ & $1.2 \pm 0.4$ & $9.3 \pm 4.2$ \\
Medications & & \\
$\quad$ Inhaled $\beta$-agonist & 4 & 4 \\
$\quad$ Inhaled corticosteroid & 3 & 3 \\
$\quad$ Oral corticosteroid & 2 & 1 \\
$\quad$ Theophylline & 2 & 2 \\
Duration of asthma yrs & $16.8 \pm 14.3$ & $15.0 \pm 8.2$ \\
Symptom frequency & & \\
Daily & 3 & 3 \\
$\quad$ Occasional & 1 & 1 \\
Hospitalization due to asthma & 2 & 3 \\
Smoking & & \\
Yes & & $0 / 3$ \\
No & $0 / 4$ & $2 / 3$ \\
Ex- & $3 / 4$ & $1 / 3$ \\
\hline
\end{tabular}

\#: mean \pm sD. M: male; F: female. ${ }^{\dagger}$ : only 3 cases available from Long duration.

\section{Inflammatory cells}

Neutrophil counts were similar in serial sections stained with either haematoxylin and eosin or with anti-neutrophil elastase (fig. 2). When all neutrophils, including those in blood vessels, that stained positively for antineutrophil elastase were counted, there were more $(\mathrm{p}<0.05)$ neutrophils in the cases of short duration $(7.1 \pm$ $\left.5.6 \mathrm{~mm} \cdot \mathrm{Pbm}^{-1}\right)$ than in the cases of long duration $(1.7 \pm 1.3$ $\left.\mathrm{mm} \cdot \mathrm{Pbm}^{-1}\right)$. Similarly, when only extravascular neutrophils stained with anti-neutrophil elastase were counted, there were more neutrophils $(\mathrm{p}<0.05)$ in the cases of short duration $\left(0.45 \pm 0.14 \mathrm{~mm} \cdot \mathrm{Pbm}^{-1}\right)$ than in cases of long duration $\left(0.27 \pm 0.09 \mathrm{~mm} \cdot \mathrm{Pbm}^{-1}\right)$. There were more extravascular neutrophils stained with haematoxylin and eosin in the short duration cases $(0.72 \pm 0.83$

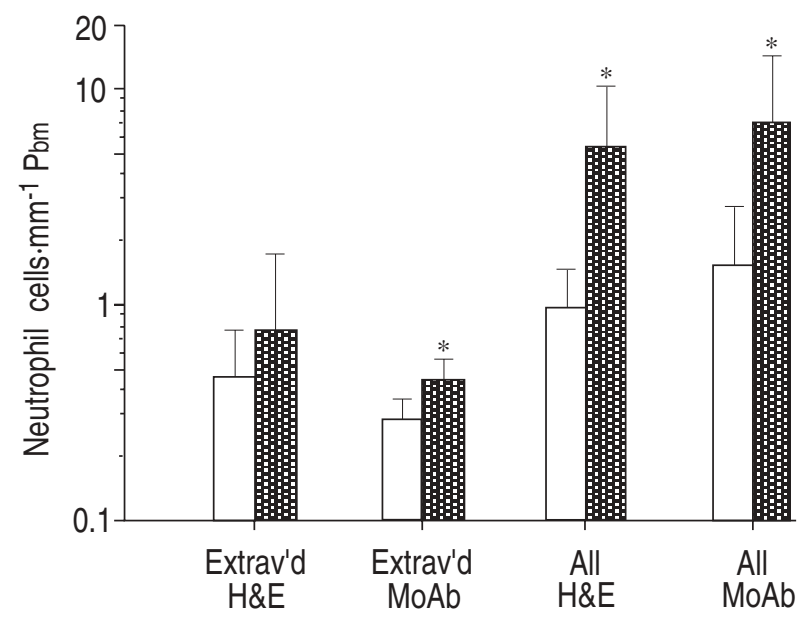

Fig. 2. - Neutrophils numbers stained with haematoxylin and eosin (H\&E) and an anti-neutrophil elastase monoclonal antibody (MoAb) in cases of short duration fatal asthma ( asthma ( $\square$ ) in cartilaginous airways $(\mathrm{Pbm}>10 \mathrm{~mm})$. Data are presented as mean $\pm \mathrm{SD}$; error bars representing $1 \mathrm{SD}$. All: inside and outside blood vessels; Extrav'd: extravasated, i.e. not in blood vessels. $\mathrm{Pbm}$ : basement membrane perimeter. $*: \mathrm{p}<0.05$, between short and long duration fatal asthma. $\left.\mathrm{mm} \cdot \mathrm{Pbm}^{-1}\right)$ than in the long duration cases $(0.48 \pm 0.26$ $\left.\mathrm{mm} \cdot \mathrm{Pbm}^{-1}\right)$, although this difference was not significant.

Figure 3 shows the eosinophil and lymphomononuclear cell counts in case groups for cartilaginous airways with $\mathrm{Pbm}>10 \mathrm{~mm}$. Neutrophil counts (as in figure 2) are included for comparison. Eosinophils in the inner wall area were increased $(\mathrm{p}<0.05)$ in cases of long duration $\left(3.5 \pm 3.0 \mathrm{~mm} \cdot \mathrm{Pbm}^{-1}\right)$ compared with cases of short duration $\left(0.93 \pm 0.90 \mathrm{~mm} \cdot \mathrm{Pbm}^{-1}\right)$. Lymphomononuclear cell were similar in the two case groups (long duration $=192 \pm 150$ $\mathrm{mm} \cdot \mathrm{Pbm}^{-1}$ versus short duration $\left.=284 \pm 261 \mathrm{~mm} \cdot \mathrm{Pbm}^{-1}\right)$.

\section{Airway dimensions and components}

Figure 4 shows the results for airway dimensions and structural components for cases of long and short duration. The basement membrane perimeters (mean $\pm \mathrm{SD})$ were

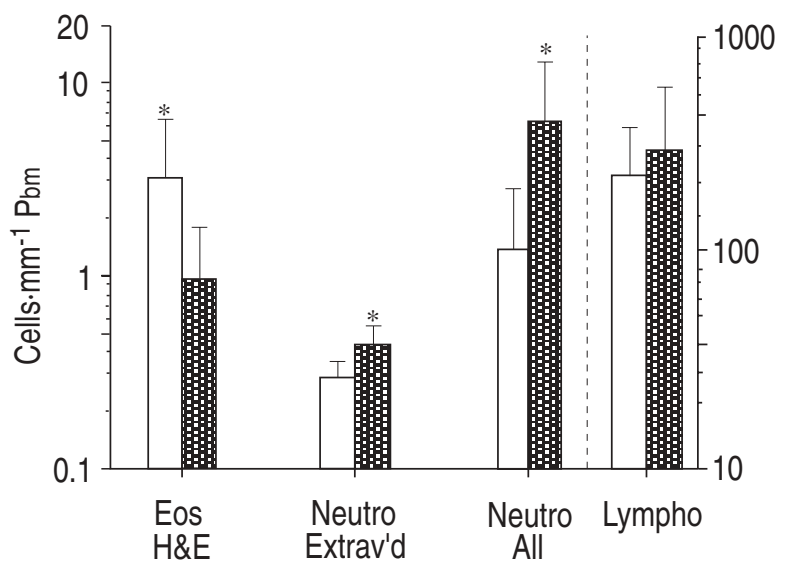

Fig. 3. - Eosinophils, neutrophils and lymphomononuclear cells in cases of short duration fatal asthma ( asthma ( $\square$ ) in cartilaginous airways $(\mathrm{Pbm}>10 \mathrm{~mm})$. Data are presented as mean $\pm \mathrm{SD}$; error bars representing $1 \mathrm{SD}$. All: inside and outside blood vessels; Extrav'd: extravasated, i.e. not in blood vessels; Pbm: basement membrane perimeter; Eos: eosinophils; Neutro: neutrophils; Lympho: lymphomononuclear cells. $*: \mathrm{p}<0.05$, between short and long duration fatal asthma.

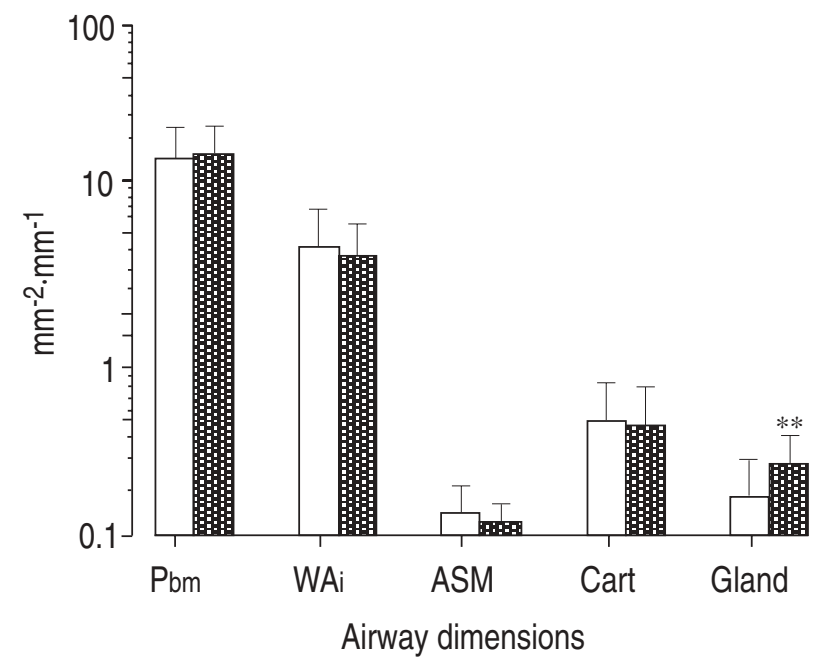

Fig. 4. - Basement membrane perimeter $(\mathrm{Pbm})$, inner airway wall area (WAi), and areas of smooth muscle (ASM), cartilage (Cart) and mucous gland (Gland) in cases of short duration fatal asthma ( and long duration fatal asthma ( $\square$ ) in cartilaginous airways ( $\mathrm{Pbm}$ $>10 \mathrm{~mm}$ ). Data are presented as mean \pm sD; error bars representing 1 SD. $* *: \mathrm{p}=0.008$, between short and lung duration fatal asthma. 


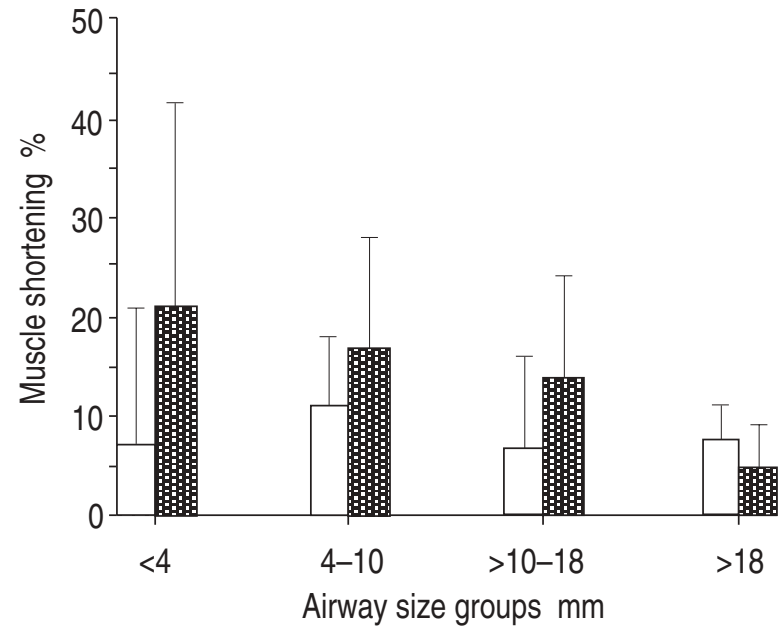

Fig. 5. - Percentage smooth muscle shortening (PMS) in cases of short duration fatal asthma (眀眲) and long duration fatal asthma $(\square)$ in four airway size groups. Data are presented as mean $\pm \mathrm{SD}$; error bars representing $1 \mathrm{SD}$.

similar in each group showing that similar sized airways were examined. The thickness of the inner airway wall (WAi) was similar in the two case groups as were the areas of airway smooth muscle and cartilage in the airway wall. The mucous gland area was greater $(\mathrm{p}=0.008)$ in the cases of short duration $\left(0.30 \pm 0.09 \mathrm{~mm}^{2} \cdot \mathrm{mm}^{-1} \mathrm{Pbm}\right)$ than in the cases of long duration $\left(0.16 \pm 0.12 \mathrm{~mm}^{2} \cdot \mathrm{mm}^{-1}\right.$ $\mathrm{Pbm})$. The calculated percentage of airway smooth muscle shortening ranged from $2-51 \%$ in different airways (fig. 5). The mean muscle shortening tended to be greater in short duration cases in all but the largest airway size group. The overall degree of muscle shortening was small and the differences between groups were not significant.

\section{Discussion}

In this study, the number of neutrophils and the area of mucous glands were increased, and the number of eosinophils was decreased, in cases with fatal asthma attacks of short duration, compared to cases with fatal attacks of long duration. Structural dimensions, the areas of airway smooth muscle and cartilage, the amount of smooth muscle shortening and the numbers of lymphomononuclear cells were similar in the two groups. All cases examined in this study were out of hospital deaths, in patients who had severe asthma requiring constant treatment and at least one hospital admission for asthma. There were no differences in neutrophil counts in the inner wall between tissue sections stained with haematoxylin and eosin and those stained with a monoclonal antibody against neutrophil elastase. The findings of this study are in agreement with those of Azzawi et al. [17] and SUR et al. [20], who reported similar cellular profiles in relationship to the duration of the final asthma attack; and extend them by showing that changes are present in airways collected prospectively with standard sampling techniques and that mucous gland enlargement is greater in the cases of short duration.

Subjects in the present study were categorized according to the time interval to death following the onset of the asthma attack. This was done with as much certainty as possible from the available Coroner's files. Information regarding control of their asthma and compliance with treatment in the hours/days leading up to the fatal episode was often unavailable or incomplete. To the best of our knowledge, all cases examined in this study were apparently well prior to the terminal episode. All cases included for examination could be considered as severe asthmatics based on symptoms, treatment and hospital admissions for asthma and duration of asthma. Based on the evidence available, we could not distinguish between the long and short duration groups with regard to severity of their asthma. It is possible that, in individuals with poorly perceived symptoms, there may be a delay between the actual onset of airway narrowing and the time course until such an individual complains of symptoms. If this were the case, then it is possible that some of the cases categorized as short duration fatal asthma may have had marked airway narrowing for some time before complaining of asthma symptoms. We had no information in this regard on any of the cases.

There are at least three possible explanations for the differences in inflammatory cell numbers between the long and the short duration cases. Firstly, asthma may be a heterogeneous disease in which severity or likelihood of sudden death is related to the type of airway inflammation that is present. SuR et al. [20] suggested that their cases of "sudden onset fatal asthma", who had greater numbers of neutrophils than those with "slow onset fatal asthma", were a subgroup of asthmatics with a type of inflammation in the airway wall which predisposed them to sudden death. Our findings do not support this hypothesis. On the basis of cases available for analysis, we arbitrarily divided our cases into two intervals of duration of their fatal attack, $<2 \mathrm{~h}$ and $>5 \mathrm{~h}$, a different categorization from SuR et al. [20] who used $<1 \mathrm{~h}$ and $>2.5 \mathrm{~h}$. Our findings are essentially the same as SuR et al. [20]. This suggests that the relative numbers of neutrophils and eosinophils are a function of time rather than being confined to a group who die within a set period of time after an attack of asthma.

Secondly, differences in airway wall structure may have affected the duration of the fatal attack of asthma in individuals exposed to similar stimuli. We have previously shown that, in these cases, airway wall thickness is increased as are areas of mucous glands and smooth muscle, compared to controls [23]. It is likely that the extensive remodelling of the airway walls seen in these cases makes them more liable to excessive airway narrowing and death when the airway smooth muscle is stimulated. In the present study, we did not observe differences in airway wall area or areas of smooth muscle or cartilage between our two case groups. However, it is possible that the increased area of mucous gland observed in the cases of short duration contributed to a rapid fatal attack of asthma.

Using the measured areas of mucous glands in the cases of fatal asthma that we have observed [23], it is possible to calculate the potential effects of mucous gland discharge, in combination with smooth muscle shortening, on airway resistance [24]. These calculations show that the degree of airway resistance that occurs in association with smooth muscle shortening would be significantly greater in patients with increased mucous gland area although, 
in the absence of smooth muscle shortening, complete discharge of mucous gland contents into the airway lumen has an insignificant effect on airway resistance. Consequently, if the discharge of mucus from mucous glands was sudden and widespread and was coupled with moderate degrees of smooth muscle shortening, then it may result in death soon after the onset of a fatal attack.

Why should differences in structure give rise to different inflammatory cell profiles in relation to duration of a fatal attack? This result might be expected if these fatal asthma attacks were due to a stimulus, which not only released mucus and contracted smooth muscle but also induced inflammation with a cellular profile that evolved over some hours. The inflammatory cell profile present in the airways at the time of death would then represent a "snapshot" in time of a dynamic inflammatory process in the airways. The fact that Azzawi et al. [17] and SuR et al. [20] were able to demonstrate a significant correlation between eosinophils and time interval to death and the similarity of their results to those in the present study supports this view. It is also supported by studies showing a change in inflammatory cell profiles in the lung in relation to time after exposure to an inflammatory stimulus [25-28]. Although measured over differing time courses, these studies showed similar patterns of cellular recruitment following an inflammatory stimulus with neutrophils tending to be the predominant cell in the early phase and a subsequent peak in eosinophil recruitment and concomitant reduction in neutrophils at a later stage. Both in humans and animals, exposure to a variety of allergenic stimuli in the laboratory is associated with a sequence of events resulting in a variable number and type of inflammatory cell being recruited to the airways.

The third explanation for our findings is that, in cases of severe asthma with extensive airway wall structural changes, exposure to a stimulus which results in a predominantly neutrophilic response is likely to cause a fatal attack of short duration, whereas a stimulus which results in a predominantly eosinophilic response is likely to cause a fatal attack of long duration. A number of studies in humans and animals have examined the inflammatory responses to inhaled stimuli [26-32] and suggest that the nature of the stimulus may determine the type of cellular response in the airways. Inhalation of ozone [26] or the occupational sensitizing agent toluene diisocyanate [29] have been associated with increased numbers of neutrophils in bronchoalveolar lavage fluid. Challenge with inhaled allergen $[30,32]$ and the inflammatory response in patients with Western red cedar asthma [33] has been associated with increased numbers of eosinophils.

However, these studies did not always examine changes in relative cell numbers over time or repeat challenges with different stimuli. HunT et al. [31] observed a marked neutrophilic response in the airways of patients challenged with allergen extract which was contaminated with endotoxin. Interestingly, when one of the same patients and six new patients were challenged with endotoxin-free allergen the response was predominantly eosinophilic, suggesting that different stimuli may result in differing cellular responses. Therefore, the different inflammatory cell profiles in the airways seen in this study may have been caused by different inflammatory stimuli. There are, however, no data examining the relationship between the magnitude of the response and the nature of the inflammatory stimulus.

There was a marked decrease in the numbers of neutrophils present in the airways of the two groups when only extravascular neutrophils were counted. This, however, does not preclude neutrophils from having an important role in the acute inflammatory response to a stimulus in these cases. Significant amounts of extracellular elastase, presumably from degranulated neutrophils, is found in lung tissue from patients with fatal asthma [34], which may lead to an underestimation of cell counts of morphologically intact neutrophils. Increased numbers of neutrophils in the blood vessels suggests increased margination and adhesion [35], which, in the presence of enhanced expression of adhesive receptors and chemotactic agents, may result in increased neutrophil recruitment. In addition, the release of products from neutrophils still in the blood stream cannot be excluded and the amount of extravascular elastase, not associated with neutrophils, was not quantitated in the present study.

REID [36] has anecdotally described patients who have died suddenly during an acute attack of asthma with airways free from inspissated mucus. Indeed, one of the cases examined in the study by SuR et al. [20] had no mucus present in the airways at autopsy. In the cases studied in our laboratory, many do not have mucus in the central airways, almost certainly due to flushing of these airways during fixation via the main bronchus. Therefore, it is difficult to exclude artefact in these cases and no carefully and prospectively collected cases with "empty airways" have been reported. This does not exclude the possibility of airway occlusion due to wall oedema and/or excessive airway smooth muscle shortening as a cause of death in asthma. Whatever the mechanisms, the findings in this study and the study by SuR et al. [20] suggest that death from asthma may be precipitated by an inflammatory stimulus, resulting in cellular infiltration, smooth muscle shortening and mucous gland discharge. The nature of the stimuli in these cases remains unclear, although the association of near fatal attacks of asthma with sensitivity and exposure to Alternaria [19], and the epidemics of asthma related to airborne soybean dust [37] and thunderstorm activity [38], are interesting in this respect. It is possible, however, that the acute, fatal stimulus occurs on a background of chronic airway inflammation and structural change and that those with the most severe structural changes, such as mucous gland hypertrophy, will have the most severe responses.

\section{References}

1. Sears MR. Epidemiological trends in bronchial asthma. In: Kaliner MA, Barnes PJ, Persson CG, eds. Asthma: Its Pathology and Treatment. New York, Marcel Dekker Inc., 1991; pp. 1-49. (Lenfant C, ed. Lung Biology in Health and Disease Vol. 49).

2. $\quad$ Sly RM. Changing asthma mortality. Ann Allergy 1994; 73: 259-268.

3. Rea HH, Scragg R, Jackson R, Beaglehole R, Fenwick J, Sutherland DC. A case-control study of deaths from asthma. Thorax 1987; 47: 833-839.

4. Sears MR, Rea HH. Patients at risk for dying of asthma: New Zealand experience. J Allergy Clin Immunol 1987; 80: 477-481. 
5. Robertson CF, Rubinfield AR, Bowes G. Deaths from asthma in Victoria: a 12 month survey. Med J Aust 1990; 152: 511-517.

6. Ryan G, Musk AW, Perera DM, Stock H, Knight JL, Hobbs MST. Risk factors for death in patients admitted to hospital with asthma: a follow-up study. Aust NZ J Med 1991; 21: 681-685.

7. Saetta M, Di Stefano A, Maestrelli P, Mapp CE, Fabbri LM. Fatal asthma. Eur Respir Rev 1993; 3 (14): 396-399.

8. Richards GN, Kolbe J, Fenwick J, Rea HH. Demographic characteristics of patients with severe life threatening asthma: comparison with asthma deaths. Thorax 1993; 48: 1105-1109.

9. Saetta M, Thiene G, Crescioli G, Fabbri LM. Fatal asthma in a young patient with severe bronchial hyperresponsiveness but stable peak flow records. Eur Respir J 1989; 2: 1008-1012.

10. Dunnill MS. The pathology of asthma with special reference to changes in the bronchial mucosa. J Clin Pathol 1960; 13: 27-33.

11. Dunnill MS, Massarella GR, Anderson JA. A comparison of the quantitative anatomy of the bronchi in normal subjects, in status asthmaticus, in chronic bronchitis, and in emphysema. Thorax 1969; 24: 176-179.

12. Cardell BS, Pearson RSB. Death in asthmatics. Thorax 1959; 14: 341-352.

13. Houston JC, De Navasquez S, Trounce JR. A clinical and pathological study of fatal cases of status asthmaticus. Histopathology 1953; 2: 407-421.

14. Cutz E, Levison H, Cooper DM. Ultrastructure of airways in children with asthma. Thorax 1978; 8: 207213.

15. Heard BE, Hossain S. Hyperplasia of bronchial muscle in asthma. J Pathol 1971; 110: 319-331.

16. Saetta M, Di Stefano AD, Rosina C, Thiene G, Fabbri LM. Quantitative structural analysis of peripheral airways and arteries in sudden fatal asthma. Am Rev Respir Dis 1991; 143: 138-143.

17. Azzawi M, Johnston PW, Majumdar S, Kay AB, Jeffery PK. T-lymphocytes and activated eosinophils in airway mucosa in fatal asthma and cystic fibrosis. Am Rev Respir Dis 1992; 145: 1477-1482.

18. James AL, Pare PD, Hogg JC. The mechanics of airway narrowing in asthma. Am Rev Respir Dis 1989; 139: 242-246.

19. O'Hollaren MT, Yunginger JW, Offord KP. Exposure to an aeroallergen as a possible precipitating factor in respiratory arrest in young patients with asthma. $N$ Engl J Med 1991; 324: 359-363.

20. Sur S, Crotty TB, Kephart GM, et al. Sudden-onset fatal asthma: a distinct entity with few eosinophils and relatively more neutrophils in the airway submucosa? Am Rev Respir Dis 1993; 148: 713-719.

21. James AL, Hogg JC, Dunn LA, Pare PD. The use of the internal perimeter to compare airway size and to calculate smooth muscle shortening. Am Rev Respir Dis 1988; 138: 235-247.

22. Carroll NG, James AL. The distribution of inflammatory cells in the bronchial tree in fatal and nonfatal cases of asthma. Am Rev Respir Dis 1993; 147 (4): A516.

23. Carroll NG, Elliot J, Morton AR, James AL. The structure of large and small airways in nonfatal and fatal asthma. Am Rev Respir Dis 1993; 147: 405-410.

24. James AJ, Carroll NG. The theoretical effects of mucous gland discharge on airway resistance in asthma. Med $J$ Aust NZ 1994; 150: 45.

25. Hulbert WC, Walker DC, Jackson A, Hogg JC. Airway permeability to horseradish peroxidase in guinea-pigs: the repair phase after injury by cigarette smoke. Am Rev Respir Dis 1981; 123: 320-326.

26. Murlas C, Roum JH. Sequence of pathological change in the airway mucosa of guinea-pigs during ozone-induced bronchial hyperreactivity. Am Rev Respir Dis 1985; 131: 314-320.

27. Renzi PM, Olivenstein R, Martin JG. Effect of dexamethasone on airway inflammation and responsiveness after antigen challenge of the rat. Am Rev Respir Dis 1993; 148: 932-939.

28. Metzger WJ, Zavala D, Richerson HB, et al. Local allergen challenge and bronchoalveolar lavage of allergic asthmatic lungs: description of the model and local airway inflammation. Am Rev Respir Dis 1987; 135: 433-440.

29. Fabbri LM, Boschetto P, Zocca E, et al. Bronchoalveolar neutrophilia during late asthmatic reactions induced by toluene diisocyanate. Am Rev Respir Dis 1987; 136: 36-42.

30. de Monchy JGR, Kauffman HF, Venge P, et al. Bronchoalveolar eosinophilia during allergen-induced late asthmatic reactions. Am Rev Respir Dis 1985; 131: 373-376.

31. Hunt LW, Gleich GJ, Ohnishi T, et al. Endotoxin contamination causes neutrophilia following pulmonary allergen challenge. AmJ Respir Crit Care Med 1994; 149: 1471-1475.

32. Rossi GA, Crimi E, Lantero S, et al. Late-phase asthmatic reaction to inhaled allergen is associated with early recruitment of eosinophils in the airways. Am Rev Respir Dis 1991; 144: 379-383.

33. Lam S, LeRiche J, Phillips D, Chan-Yeung M. Cellular and protein changes in bronchial lavage fluid after late asthmatic reaction in patients with Western red cedar asthma. J Allergy Clin Immunol 1987; 80: 44-50.

34. Fujisawa T, Kephart GM, Gray BH, Gleich GJ. The neutrophil and chronic allergic inflammation: immunochemical localization of neutrophil elastase. Am Rev Respir Dis 1990; 141: 689-697.

35. MacNee W, Selby C. Neutrophil traffic in the lungs: role of haemodynamics, cell adhesion and deformability. Thorax 1993; 48: 79-88.

36. Reid LM. The presence or absence of bronchial mucus in fatal asthma. J Allergy Clin Immunol 1987; 80: 415-416.

37. Anto JM, Sunyer J, Reed CE, et al. Preventing asthma epidemics due to soybeans by dust control measures. $N$ Engl J Med 1993; 329 (24):1807-1808.

38. Bellomo R, Gigliotti P, Treloar A, et al. Two consecutive thunderstorm associated epidemics of asthma in the city of Melbourne: the possible role of rye grass pollen. Med J Aust 1992; 156: 834-837. 